\title{
The lightning-rod mode in a core shell nanocylinder dimer
}

\author{
J.Y. Lu* ${ }^{*}$ Y.H. Chang \\ Department of Physics, National Taiwan University, Taipei, Taiwan
}

\section{A R T I C L E I N F O}

\section{Article history:}

Received 13 November 2009

Received in revised form 10 February 2010

Accepted 10 February 2010

Available online $\mathrm{xxxx}$

\begin{abstract}
A B S T R A C T
Optical properties of a dielectric-core gold-shell nanocylinder pair are investigated by two-dimensional finite difference time domain method. The results show that the plasmonic interaction between the two dielectric-core gold-shell nanocylinders could contain an additional plasmon mode. This additional plasmon mode is resulted from the coupling of the electron at the outer surfaces of the two nanocylinders due to gold shell and gold/dielectric boundary that block electromagnetic wave. Depending on the permittivity of the dielectric core and shell thickness of the nanocylinders, we can decide the existence of this additional plasmon mode in the spectrum.
\end{abstract}

(c) 2010 Elsevier B.V. All rights reserved.

\section{Introduction}

Optical properties of metallic nanostructures are attractive because of their ability to enhance, emit, and modify the electromagnetic field. Recent advances in nanofabrication have enabled us to design nanostructures with different shapes and functionalities, such as nanorices [1], nanorings [2] nanoshells [3], and the dependence of the optical properties of these metallic nanostructures on their sizes, shapes, and surrounding media has been a subject of active research.

The plasmon modes of a solid nanostructure are characterized by the surface charge distributions on the surface of the solid nanostructure [4]. For the core-shell structures, the interaction between the surface charge distributions at the inner and outer surfaces of the core-shell structure splits the plasmon resonance modes into two different alignment plasmon modes and result in the large variation of the plasmon resonance wavelength for the core-shell nanocylinder. The plasmon resonance wavelength of the core-shell structure can be tuned by the permittivity of the dielectric core and the ratio of the outer radius to inner radius [5]. By the same token, when two plasmon-resonant core-shell nanoparticles are brought together, the plasmon modes in the individual nanocylinders can interact with each other, leading to new resonance modes for the coupled system. These plasmon modes have been investigated in detail in [3,6-9].

In this work, a new plasmon mode associated with the core-shell nanocylinder pair is reported. We found that a resonance mode resulted solely from the coupling of the electrons at the outer surfaces of the two core-shell nanocylinders can be observed when the size of the nanocylinder is large enough. The

\footnotetext{
* Corresponding author.

E-mail address: f95222042@ntu.edu.tw (J.Y. Lu).
}

size-dependence and permittivity-dependence of this special plasmon mode indicate that this plasmon mode shows up as a result of the screening effect of the metallic shell and reflection at the metal/dielectric boundary. Such a phenomenon is essentially caused by the lightning-rod effect, which occurs when metals approach to the perfect conductors in low frequency and the electric field concentrates in the gap of the nanocylinder dimer. A related discussion of plasmonic systems associated with the lightning-rod effect can be found in [15].

\section{Calculation methods}

Our finite difference time domain (FDTD) simulation domain is separated into three regions from outside to inside: absorbing boundary, scattered field region, and the total field region. The perfectly matched layers (PMLs) are used as absorption boundary to prevent reflections of scattered waves back into the simulation domain. The FDTD calculations were done accurately using a mesh size of $0.25 \mathrm{~nm}$, a time step of $4.16666 \mathrm{e}-19 \mathrm{~s}$, and a Courant number of 0.5 . The program codes have been checked with the analytical theory [10]. The optical response of gold is modeled using the three critical point pole pairs (CP3) model [11] which provides a good fit to tabulated experimental data [12]. The geometrical arrangement of the nanocylinder pair and the incident wave is shown in Fig. 1, the propagating direction of the incident wave is perpendicular to the axis that connects the nanocylinder pair, and the electric field of the incident wave is parallel to this axis.

\section{Results and discussion}

We first investigate the extinction spectra of a silica-core goldshell nanocylinder pair, for different sizes. The ratio of outer radius to inner radius is fixed at $6: 5$, the ratio of outer radius to separation 


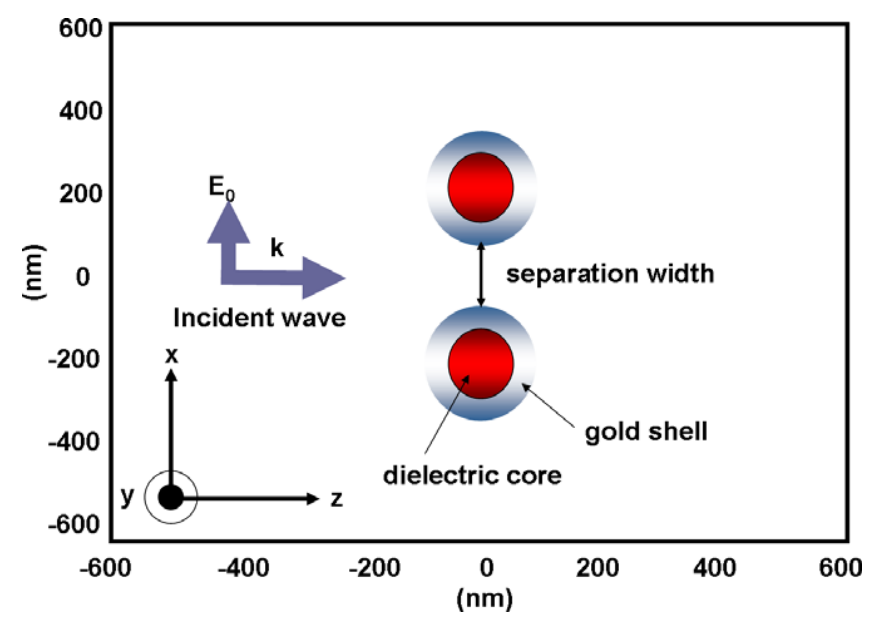

Fig. 1. Schematic diagram of the geometrical arrangement of the dielectric-core gold-shell nanocylinder pair.

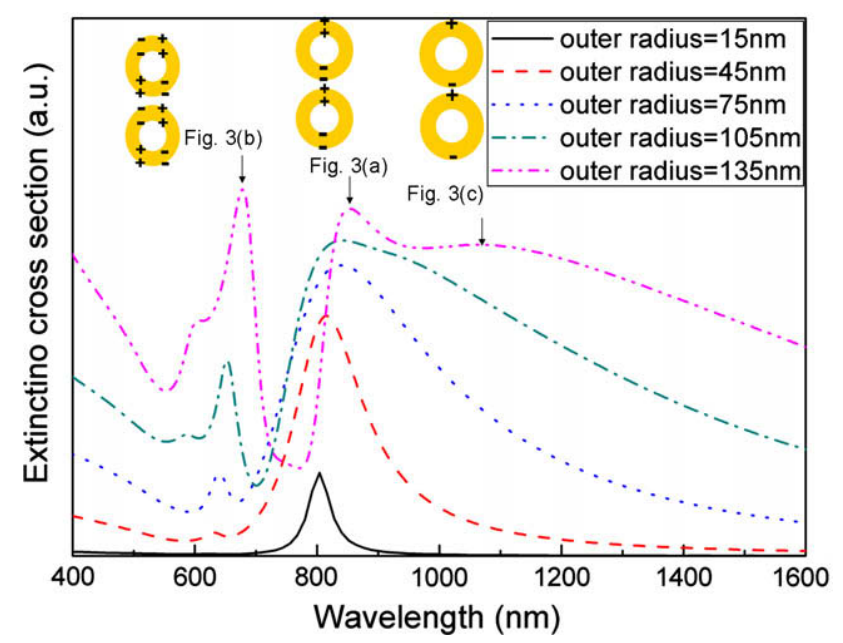

Fig. 2. The extinction spectra as a function of size of the silica-core gold-shell nanocylinder pair from the outer radius of $15-135 \mathrm{~nm}$. The ratio of the outer radius to inner radius is fixed at $6: 5$ and the ratio of the outer radius to the internanocylinders spacing at $5: 1$.

distance between two nanocylinders is fixed at 5:1, and the outer radius is varied from $15 \mathrm{~nm}$ to $135 \mathrm{~nm}$ with an interval of $30 \mathrm{~nm}$. The extinction spectra as a function of the size of the nanocylinder pair, and the charge distribution of the plasmon modes in the nanocylinders are shown in Fig. 2. For outer radius of $15 \mathrm{~nm}$, the plasmon mode, near $800 \mathrm{~nm}$, corresponds to the in-phase symmetric dipolar (SD) mode. As the size increases, because of the phase retardation effect, the resonance wavelength of the SD mode also increases. There are other two peaks at shorter wavelength in the spectra which are the in-phase symmetric quadrupolar (SQ) mode and the in-phase symmetric octopolar (SO) mode. We cannot observe the out-of-phase plasmon modes in the spectrum because the out-of-phase plasmon modes have a smaller total dipole moment than that of the in-phase plasmon modes and thus are less likely to be excited by light. In addition to these plasmon modes discussed above, we can see in Fig. 2 that as the outer radius on the nanocylinder increases to $135 \mathrm{~nm}$, an additional in-phase dipolar plasmon mode shows up near $1085 \mathrm{~nm}$.

The origin of this new plasmon mode is studied by finding the electric field intensity of the silica-core gold-shell nanocylinder pair with outer radius of $135 \mathrm{~nm}$ and the results are shown in
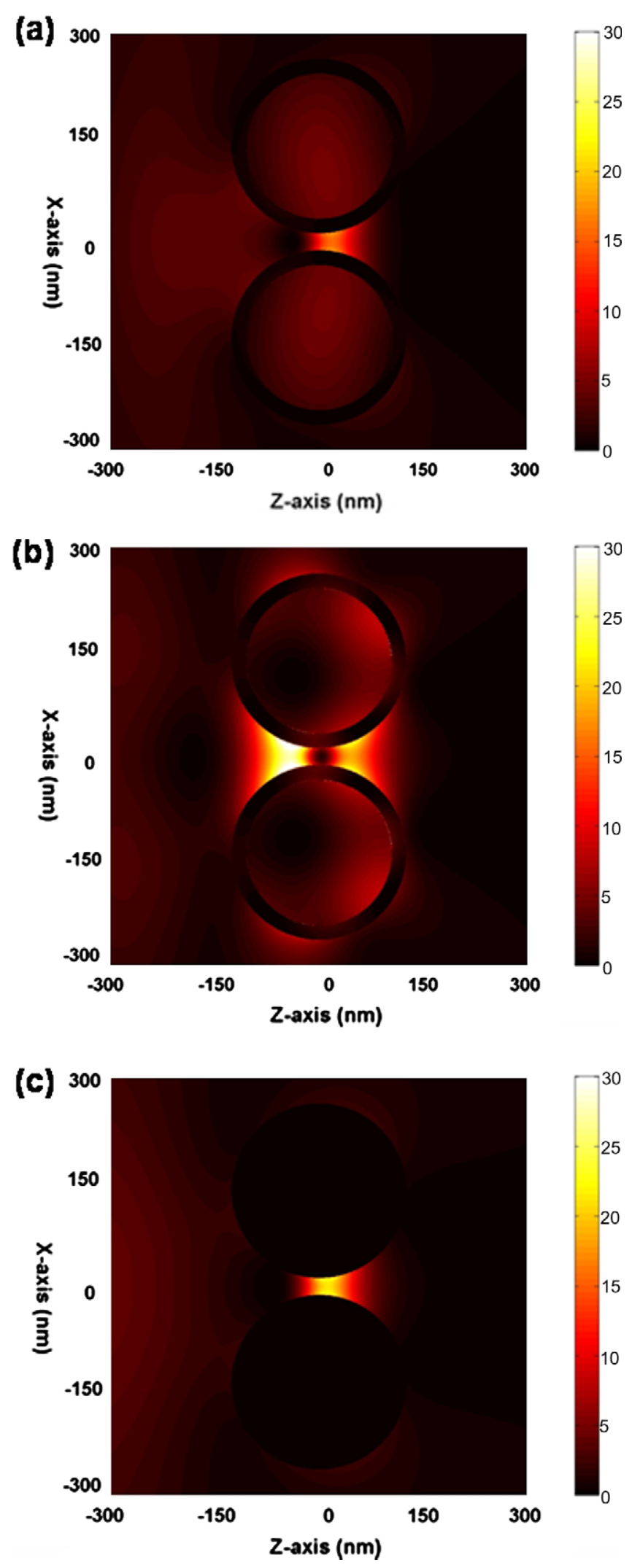

Fig. 3. The extinction spectra as a function of size of a pure solid gold nanocylinder pair from the radius of $45-135 \mathrm{~nm}$. The ratio of the radius to the inter-nanocylinders spacing is fixed at 5:1.

Fig. 3. In these figures, the normalized electric field intensity distributions of the core-shell nanocylinder system are found by propagating plane waves at their respective plasmon resonance 
wavelengths. The results show that for the resonances that occur at $650 \mathrm{~nm}$ (Fig. 3a) and $850 \mathrm{~nm}$ (Fig. 3b), there is a non-uniform electric field intensity distribution inside the dielectric core. The nonuniform electric field intensity is due to the formation of the cavity modes inside the dielectric core, which implies there is a non-zero charge distribution in the inner surface of the gold shell of the nanocylinder. However, for the electric field intensity of the plasmon mode near $1085 \mathrm{~nm}$ shown in Fig. 4c, we can find that the amplitude of the electric field inside the dielectric core is zero. This implies that for this resonance mode, there are no induced charges at inner surface of the gold shell in the nanocylinder. We can thus infer that this special plasmon mode is resulted from the coupling of the electrons at outer surfaces between the two core-shell nanocylinders, similar to the coupling of surface plasmon modes between a pair of solid gold nanocylinders. This conclusion is also supported by the extinction spectra (shown in Fig. 4) obtained for a pair of solid gold nanocylinders with the same diameters as the outer diameter of the core-shell nanocylinders. We can see that resonance wavelength of both the solid gold nanocylinder pair and the core-shell nanocylinder pair occur at $1085 \mathrm{~nm}$ when the outer diameter of the nanocylinder is $135 \mathrm{~nm}$. These results indicate that the appearance of this resonance mode is due to the metallic screening effect. As the resonance wavelength appear near the wavelength larger than that of known plasmon modes in the core shell nanocylinder pair, this plasmon mode can be observed in the spectrum. Because the skin depth of the gold near the resonance wavelength of $1085 \mathrm{~nm}$ is small, the electric field is completely screened by the metal, no electric field, and no charge could be found in the inner side of the metallic shell.

In the following sections we will proof this special plasmon mode solely depends on the electrons at the outer surfaces of the nanocylinder pair by varying the dielectric core and shell thickness. The dependence of this plasmon mode on the permittivity of the dielectric core is studied in this section. In Fig. 5, we study the spectral characteristics of a dielectric-core gold-shell nanocylinder pair with outer radius of $135 \mathrm{~nm}$ and inner radius of $112.5 \mathrm{~nm}$, for dielectric cores with permittivities of 2.1, 2.6, 3.3, and 4.1 , respectively. The simulation results show that except for the plasmon mode near $1080 \mathrm{~nm}$, the resonance wavelengths of the other plasmon modes are red-shifted with increasing the permittivity of the dielectric core [14]. When the permittivity of the dielectric core is larger than 3.3, the plasmon mode resulted from

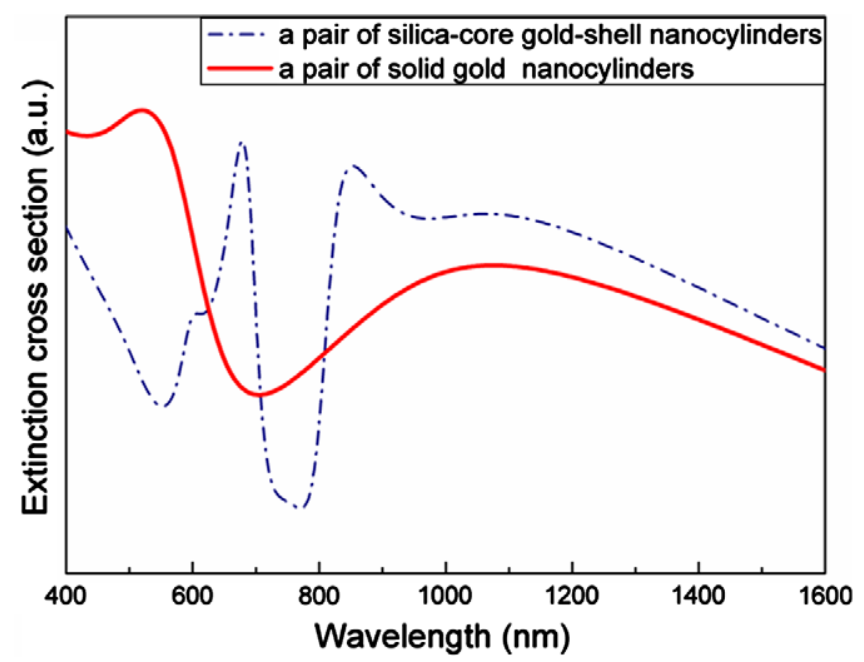

Fig. 4. The normalized electric field intensity at the respective coupling plasmon modes wavelength of a silica-core gold-shell nanocylinder with outer radius of $135 \mathrm{~nm}$ and inner radius of $112.5 \mathrm{~nm}$. Wavelengths of the incident light are (a) $851 \mathrm{~nm}$, (b) $680 \mathrm{~nm}$, and (c) $1085 \mathrm{~nm}$.

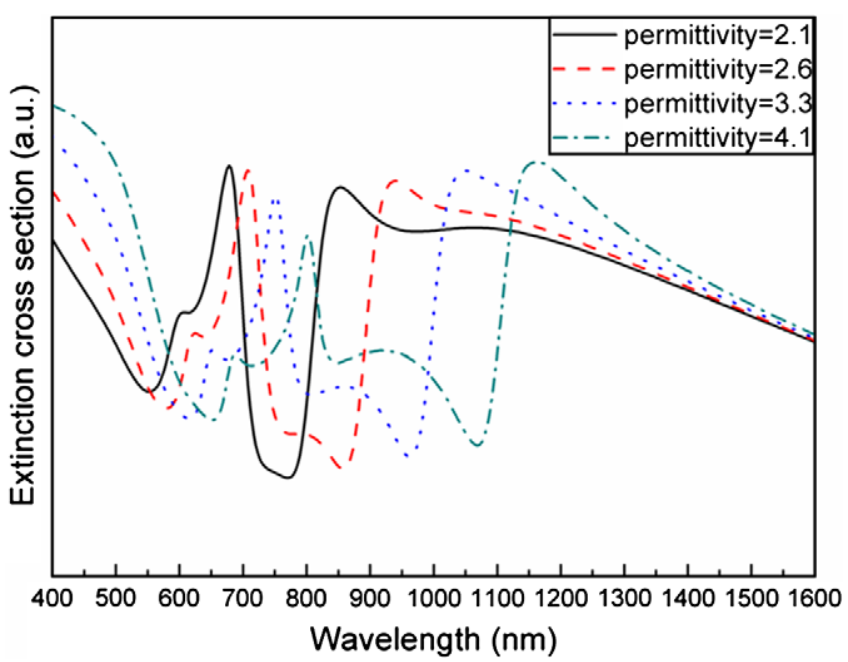

Fig. 5. The dependence of the extinction spectra of the nanocylinder pair with outer radius of $135 \mathrm{~nm}$ and inner radius of $112.5 \mathrm{~nm}$ on the permittivity of the dielectric core.

the outer surface charges near $1085 \mathrm{~nm}$ disappears. For comparably large dielectric-core gold-shell system, the symmetric dipolar (SD) mode inside the nanocylinder has the cavity resonance nature, which is similar to one-dimensional Fabry-Pérot resonance [13]. A higher permittivity reduces the reflectance at the boundary of gold/dielectric due to a closer match in the magnitude of the real part of the dielectric constant and leads to reduce the blocking EM wave effect. Therefore, as the permittivity of the dielectric core increases, this plasmon mode disappears because the light can penetrate through the metallic shell.

We can also observe that as the permittivity of the dielectric core increases, an interesting weak plasmon mode near $924 \mathrm{~nm}$ appears in the spectra. The weak plasmon mode corresponds to inphase antisymmetric dipolar plasmon mode whose antisymmetric dipolar mode has an antisymmetric alignment of the inner and outer dipolar mode of the core-shell nanocylinder.

The existence of this plasmon mode in the extinction spectrum can be also controlled by varying the thickness of the metallic layer as well as the radius of the dielectric core. In order to observe this plasmon mode more clearly, we are able to enhance the screening effect and this can be achieved by increasing the thickness of the metallic shell. In Fig. 6a, the spectra were taken with the inner radius fixed at $112.5 \mathrm{~nm}$, the inter-nanocylinder separation distance fixed at $27 \mathrm{~nm}$, and the outer radius varied from $135 \mathrm{~nm}$ to $165 \mathrm{~nm}$ with an interval $10 \mathrm{~nm}$ (corresponding to the thickness of the metallic shell from $22.5 \mathrm{~nm}$ to $52.5 \mathrm{~nm}$ ). We can see clearly the existence of the resonance mode at $1085 \mathrm{~nm}$ in this figure when the thickness of the metallic shell increases. This resonance mode can also be made to disappear by changing the cavity confinement effect. The dependence of the extinction spectra of the nanocylinder pair with the dielectric core permittivity 2.1 , the outer radius $135 \mathrm{~nm}$, the inter-nanocylinder separation distance $27 \mathrm{~nm}$, and the inner radius varied from $117.5 \mathrm{~nm}$ to $125 \mathrm{~nm}$ on core-shell dimensions is shown in Fig. 6b. As the in-phase symmetric dipolar mode is broadened and shift to longer wavelength due to less cavity confinement, the plasmon mode resulted from the coupling of the electrons at outer surfaces of the nanocylinder pair disappear when the resonance wavelength of the in-phase symmetric dipolar mode is larger than its resonance wavelength. In addition to the permittivity of the dielectric core and the thickness of the gold shell, we can also tune the plasmon resonance modes by varying the separation distance between the two nanocylinders. The dependence of the extinction spectra of the nanocylinder pair with the dielectric 

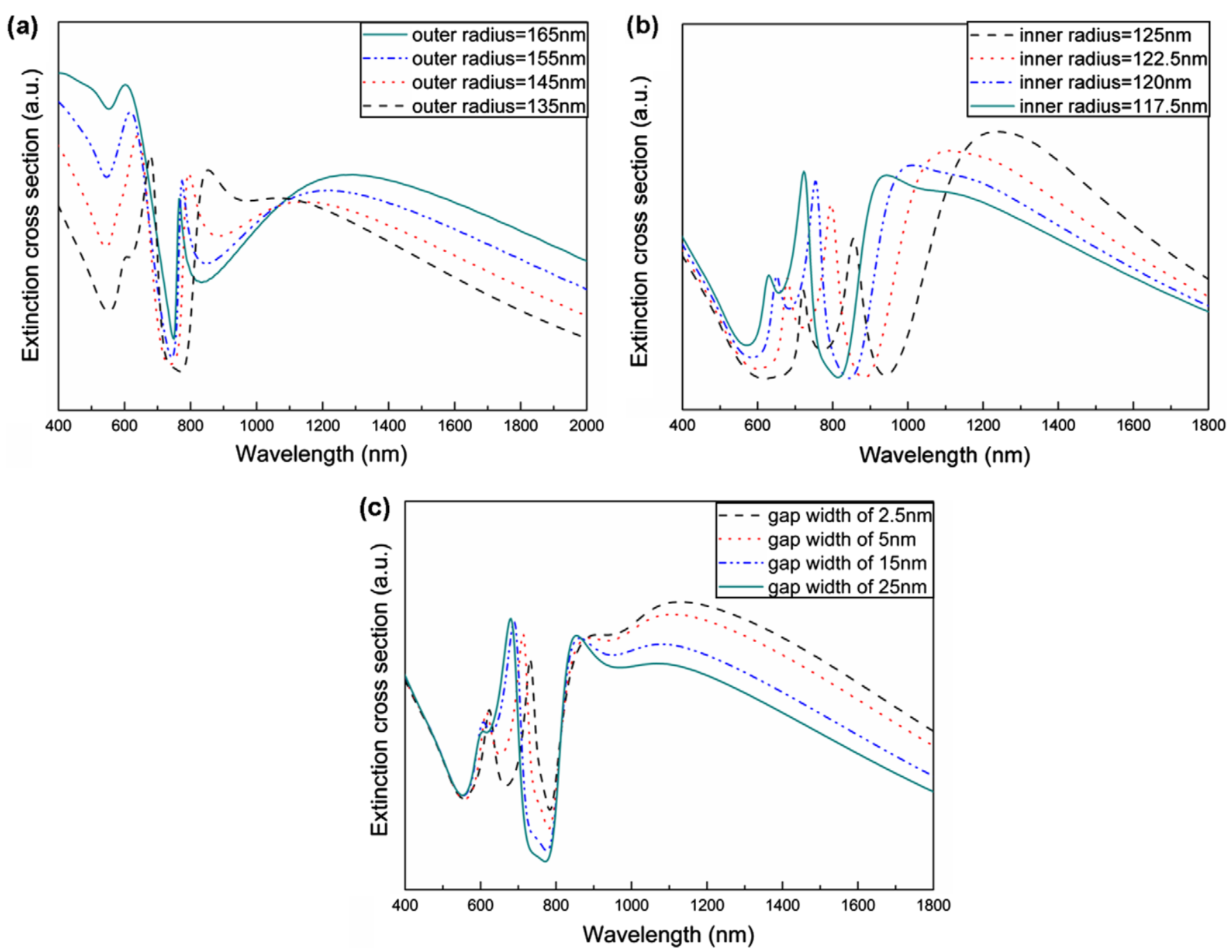

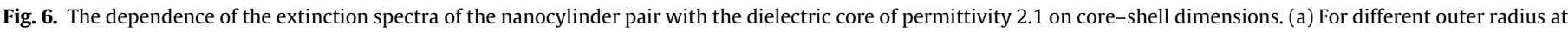

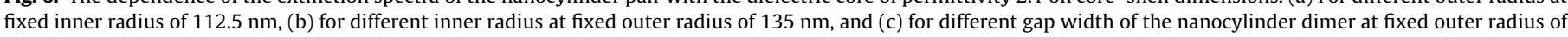
$135 \mathrm{~nm}$ and inner radius of $112.5 \mathrm{~nm}$.

core of permittivity 2.1 , the outer radius of $135 \mathrm{~nm}$, the inner radius of $112.5 \mathrm{~nm}$, and the gap width between the nanocylinder pair varied from $25 \mathrm{~nm}$ to $2.5 \mathrm{~nm}$ is shown in Fig. 6c. As the gap width between the nanocylinder pair decreases, the screening plasmon mode is stronger and red-shifted due to the larger coupling between the outer surfaces of the two nanocylinders.

\section{Conclusion}

In conclusion, we have shown that the plasmon modes of a dielectric-core gold-shell nanocylinder pair have an in-phase dipolar plasmon mode whose dipolar mode is from the electrons at outer surfaces of the nanocylinder pair. As its plasmon resonance wavelength is larger than others, it can be observed in the spectrum due to EM wave blocking by the gold shell and the gold/ dielectric boundary. Therefore, we can control the existence of this plasmon mode by changing the shell thickness and the permittivity of the dielectric core. We expect that our findings can improve let us have a better understanding of the plasmonic properties and enable us to have better ability for designing a device tailored for specific applications such as surface enhanced Raman scattering (SERS), where one wants to maximize the field enhancement over a specific frequency range.

\section{Acknowledgment}

We are grateful to the National Center for High-performance Computing for computer time and facilities.

\section{References}

[1] H. Wang, D.W. Brandl, F. Le, P. Norlander, N.J. Halas, Nano Lett. 6 (4) (2006) 827.

[2] J. Aizpurua, P. Hanarp, D.S. Sutherland, M. Kall, G.W. Nryant, F.J. Garcis de Abajo, Phys. Rev. Lett. 90 (2003) 057401.

[3] J.B. Lassiter, J. Aizpurua, L.I. Hernandez, D.W. Brandl, I. Romero, S. Lal, J.H. Hafner, P. Nordlander, N.J. Halas, Nano Lett. 8 (2008) 1212.

[4] H. Wang, Y. Wu, B. Lassiter, C.L. Nehl, J.H. Hafner, P. Norlander, N. Halas, Proc. Natl. Acad. Sci. USA. 103 (2006) 10856

[5] E. Prodan, C. Radloff, N.J. Halas, P. Norlander, Science 302 (2003) 419.

[6] D.W. Brandl, C. Oubre, P. Norlander, J. Phys. Chem. B 123 (2005) 024701.

[7] C. Oubre, P. Norlander, J. Phys. Chem. B 108 (2004) 17740.

[8] J.W. Liaw, J.H. Chen, C.S. Chen, M.K. Kuo, Opt. Express 17 (2009) 13534.

[9] A.O. Pinchuk, G.C. Schatz, Appl. Phys. B 93 (2008) 31

[10] H.A. Yousif, R.E. Mattis, K. Kozminski, Appl. Opt. 33 (1994) 4013.

[11] J.Y. Lu, Y.H. Chang, Superlatt. Microstruct. 47 (2010) 60.

[12] P. Johnson, R. Christy, Phys. Rev. B 6 (1972) 4370.

[13] J.J. Penninkhof, A. Moroz, A.V. Blaaderen, A. Polman, J. Phys. Chem. C 112 (2008) 4146.

[14] N.K. Grady, N.J. Halas, P. Nordlander, Chem. Phys. Lett. 399 (2004) 167.

[15] F. Le, D.W. Brandl, Y.A. Urzhumov, H. Wang, J. Kundu, N.J. Halas, J. Aizpurua, P. Nordlander, ACS Nano 2 (2008) 707. 